\title{
Expression of PD-L1 for predicting response to immune checkpoint inhibitors in metastatic urothelial carcinoma: a systematic review and meta-analysis
}

\author{
J. Huang $\mathrm{MD}^{*}$ and $\mathrm{X}$. Teng $\mathrm{MD}^{\dagger}$
}

\begin{abstract}
Background We conducted this meta-analysis and systematic literature review to study the ability of PD-L1 to predict objective response in patients with urothelial cancer treated with PD-1/PD-L1 inhibitors.

Methods Relevant studies of PD-1 or PD-L1 inhibitors in urothelial cancer that reported objective response rate (ORR) based on PD-L1 expression status in PubMed, EMBASE, and the Cochrane Library were retrieved. Efficacy of PD-L1 expression status in predicting ORR and the efficacy, safety of PD-1 and PD-L1 drugs were analyzed.

Results Studies were divided into $\geq 1 \%, \geq 5 \%$, and $\geq 25 \%$ based on PD-L1 positivity threshold, and the patients were grouped into PD-L1 positive and negative. In all 3 expression thresholds, patients with positive PD-L1 expression were more likely to experience an objective response [ $\geq 1 \%$ threshold odds ratio (OR): $1.74 ; 95 \%$ confidence interval (CI): 1.20 to 2.53 ; $\geq 5 \%$ threshold OR: $2.74 ; 95 \%$ CI: 2.01 to $3.724 ; \geq 25 \%$ threshold OR: 7.13 ; $95 \%$ CI: 2.38 to 21.40 ] in comparison with patients with negative PD-Ll expression. Of the 3 thresholds, the $\geq 25 \%$ threshold was better in predicting ORR (1.74 vs. 2.93 vs. $7.13 ; p<0.0001$ ). The $\geq 1 \%$ PD-L1 threshold had a relatively high sensitivity in predicting ORR; the $\geq 5 \%$ PD-L1 threshold was better for specificity. Sensitivity was higher at the $\geq 25 \%$ threshold than at the other two thresholds, but specificity was lower. Further, we found that there is no statistically significant difference in efficacy between PD-1 and PD-Ll drugs.
\end{abstract}

Conclusions Urothelial cancer patients with PD-L1 positive expression responded better than PD-L1 negative patients did, and a threshold of $\geq 5 \%$ or greater for PD-L1 expression might predict positive clinical response.

Key Words Meta-analyses, PD-1, biomarkers, atezolizumab, pembrolizumab

Curr Oncol. 2020 December:27(6)e656-e663

www.current-oncology.com

\section{INTRODUCTION}

Among all malignancies, urothelial carcinomas are paradoxical in their predilection for the developed world, where their incidence is higher than in the rest of the world ${ }^{1}$. The conventional treatment options available for urothelial cancer include radical cystectomy and neoadjuvant chemotherapy, including platinum-based cisplatin treatment. The therapeutic choice depends on whether the malignancy is invasive, noninvasive, or metastatic. The therapeutic armamentarium against urothelial cancer is further reduced in cisplatin-ineligible patients, for whom the therapeutic options are limited ${ }^{2}$.

Currently there are 5 immunotherapy-based drugs belonging to the PD-1/PD-L1 class approved by the U.S. Food and Drug Administration (FDA) for urothelial cancer (pembrolizumab, atezolizumab, nivolumab, durvalumab, and avelumab). The approvals were based on phase I/II trials for cisplatin-ineligible patients and as second-line therapy ${ }^{3-7}$. These drugs are monoclonal antibodies targeting PD-1/PDLl, which play a role in conferring antitumour immunity. Cells in the tumour microenvironment-tumour cells and 
immune cells alike-express PD-1 and PD-L1 that activates the immune checkpoint pathway, thereby evading the immune response ${ }^{8}$. PD-L1 is one of the many ligands activating the $\mathrm{PD}-1$ receptor. Because $\mathrm{PD}-1$ could also be activated by other ligands, such as PD-L2, leading to activation of the immune checkpoint pathway, it is presumed that PD-1 inhibitors might be associated with better efficacy than PD-L1 inhibitors ${ }^{9}$. Owing to the fact that PD-L1 is expressed in multiple cell types, there is no consensus about the cell population to be used for PD-L1 expression. Further, the expression threshold defining positive and negative PD-L1 expression is also widely debated. Different diagnostic kits prescribe either the tumour cells or the immune cells in the tumour microenvironment or any of the two cell populations for estimating PD-L1 expression. The threshold level also varies from $\geq 1 \%$ to $\geq 25 \%$ for defining positive PD-Ll expression.

Although durable responses were observed in early PD-1/PD-L1 trials, the response rates ranged from $10 \%$ to $30 \%$ in various malignancies indicating that only a subset of patients might derive benefit from PD-1/PD-L1 drugs ${ }^{10,11}$. This prompted investigators to look for prospective biomarkers for which PD-L1 seemed to be a rational choice. To facilitate PD-L1 expression-guided pharmacotherapy, FDA-approved immunohistochemistry-based diagnosis of PD-L1 expression levels as a companion or complementary diagnostic assay for various types of malignancies including urothelial cancer are to be used in conjunction with specific PD-1/PD-L1 drugs ${ }^{12-16}$.

Recent results from phase III trials with pembrolizumab and atezolizumab report contradictory findings ${ }^{16,17}$. There could be several reasons for the observed discordance between phase I/II trials and phase III trials. The foremost reason could be that the assays were developed independent of each other, which might introduce variations that could have been compounded by patientspecific factors. The methodologic variations were further amplified by the different PD-L1 thresholds and diagnostic kits used for the various malignancies. In light of the new phase III results, it is essential to revisit the initial evidence favouring PD-L1 expression as a biomarker for selecting urothelial cancer patients likely to benefit from PD-1/ PD-L1 drugs. Also, phase III trial results were available only for atezolizumab and pembrolizumab. We therefore performed a meta-analysis to explore the prognostic utility of PD-L1 expression in predicting therapeutic response and to compare the efficacy and safety of PD-L1 and PD-1 drugs in urothelial cancer.

\section{METHODS}

This meta-analysis was performed in accordance with the PRISMA (Preferred Reporting Items for Systematic Reviews and Meta-Analyses)) statement guidelines ${ }^{22}$. The study was registered in PROSPERO with registration number CRD42018115545.

\section{Literature Search and Selection}

We performed a comprehensive systematic search of PubMed, EMBASE, and the Cochrane Library from inception to October 2018 without language restrictions. The search strategy included the following key words: "urothelial carcinoma" OR "urothelial cancer" OR "urothelial tumour" AND "nivolumab" OR "atezolizumab" OR "pembrolizumab" OR "avelumab" OR “durvalumab” OR "avelumab.” The inclusion and exclusion criteria were prespecified. To be eligible for inclusion, the studies had to be clinical trials done in urothelial cancer patients treated with any of the PD-1/PD-L1 inhibitors either as first-line or second-line treatment with objective response rate (ORR) as the primary or secondary outcome. Further, PD-L1 expression status and PD-L1 expression-stratified ORR data had to be provided in the study for inclusion. Publications other than clinical trials, such as reviews, commentaries, meta-analyses, editorials, cost-effectiveness analyses, and observational cohort studies, were excluded. Trials evaluating a combination of PD-1/PD-L1 and other standard-of-care drugs were also excluded. If the same cohort of patients was used in multiple studies, only the study with the updated or comprehensive data was included for analysis. A secondary search was also conducted in the reference list of all the included articles for relevant studies missed in the initial search. The relevant studies were screened by two independent reviewers, and disparities were settled by a third independent reviewer.

\section{Methodologic Quality Assessment}

Methodologic quality of the included studies was evaluated using the Newcastle-Ottawa scale for cohort studies given that the retrieved studies were single-arm trials. We evaluated and rated the included studies based on the patient selection criteria and outcome assessment. We considered a study with a score of 5 or greater to be a high-quality study given that a standard criterion for what constitutes a high-quality study has not yet been universally established for this scale.

\section{Data Extraction and Outcome Measures}

Data extraction was performed based on 5 different parameters:

Demographic details, including age, sex, median duration of treatment, and mean follow-up

PD-L1 expression testing methods (antibody clones, assay developers, interpretation of test results, and the threshold for positivity

Efficacy outcomes, including ORR, median progressionfree survival (mPFs), and median overall survival (mos) Safety outcomes, with the number and types of adverse events

Efficacy, based on PD-L1 expression status

The PD-L1 expression testing method was also included in the analysis to explore the impact of the testing platform on overall results. Two investigators independently reviewed data retrieved from each article. Discrepancies between reviewers was resolved by discussion and consensus. The level or percentage of missing data for the primary endpoint and the reasons for missing data for the various therapies was determined for each study. In the case of studies with limited data, the proportion of captured values was reported and used for analysis. 
The overall ORR, mPFs, and mos provided in each included study were used for comparing the efficacy of PD-1 and PD-L1 drugs. The number and type of adverse event were also captured for safety profile analysis. Objective response rate, stratified based on PD-L1 expression, was used for biomarker analysis and to determine the PD-L1 threshold associated with better ORR.

\section{Subgroup Analysis}

Given that multiple testing platforms and antibodies were used for PD-L1 detection, we performed a subgroup analysis for the Ventana (Roche Diagnostics, Risch-Rotkreuz, Switzerland) and Dako (Agilent Technologies, Santa Clara, CA, U.S.A.) platforms, with different thresholds to rule out the influence of the testing platform on the overall analysis.

\section{Statistical Analysis}

Due to the non-availability of os and PFs data, the primary endpoint was ORR in patients who were PD-L1 positive and PD-L1 negative, measured by odds ratio (OR). Further, 95\% confidence intervals (CIS) were calculated for the derived OR. Heterogeneity across different studies according to the reported ORR was used to calculate the $I^{2}$ statistic. A fixed-effects model was used if the $I^{2}$ was less than $50 \%$, and a random-effects model was used if the $I^{2}$ was $50 \%$ or greater. Potential publication bias was analyzed using funnel plots. All the analyses were performed using the $\mathrm{R}$ software application (version 3.4.1: R Foundation for Statistical Computing, Vienna, Austria).

\section{RESULTS}

\section{Literature Search}

The literature search retrieved 1029 publications. After removing the duplicates, 798 studies were screened for relevancy, of which 30 were considered relevant. After taking into consideration the desired outcome and study type, ten single-arm studies were included for final analysis. A detailed flowchart of the literature screening and the selection criteria is provided in Figure 1. Of the ten studies, four studies were done with PD-1 drugs and six studies were done with PD-L1 drugs. Table I summarizes the included studies.

\section{Assessment of Methodologic Quality}

Methodologic quality assessment using the NewcastleOttawa scale for cohort studies revealed nine studies to be of high quality with a score 5 or greater. Further, among the ten studies, the outcome (ORR) was investigatorassessed in three studies and was investigated by an independent review committee in the others. Because the included studies were single-arm studies, the maximum score was considered to be 6 (excluding comparability and selection of unexposed cohort). Since $77 \%$ (7/9) was used as a cut-off for high quality in previous studies ${ }^{19}$ utilizing this scale, we used 5/ 6 as the cut-off for high quality. The quality assessment scoring of each study is provided in Table I.

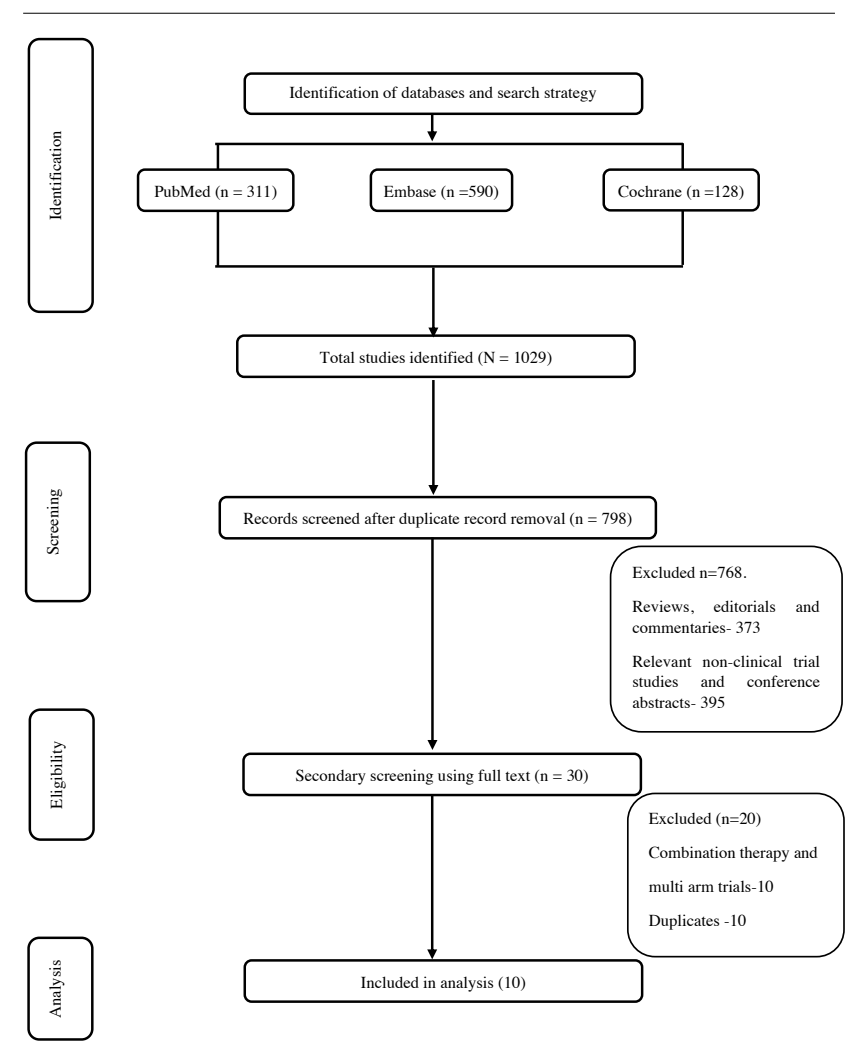

FIGURE 1 Study selection flowchart.

\section{Study Characteristics}

All the included studies were either phase I or phase II studies. In total, 1933 patients from ten clinical trials were included in this study, out of which PD-L1 expression status was determined for 1400 patients (72\%). The cell population analyzed for determining PD-L1 positivity in the included studies was either the immune cells or the tumour cells or both in the tumour microenvironment. The various thresholds used for determining PD-L1 positivity were $\geq 1 \%, \geq 5 \%, \geq 10 \%$, and $\geq 25 \%$ in different studies. Based on the threshold, the studies were grouped into $\geq 1 \%, \geq 5 \%$, and $\geq 25 \%$ for further analysis. Four studies used two different thresholds $(\geq 1 \%$ and $5 \%$ for 3 studies and $\geq 1 \%$ and $10 \%$ for 1 study) for determining PD-L1 positivity. The prevalence of PD-L1 positivity, antibody clone, and platform used for PD-L1 assay is provided in Table II. The prevalence of PD-L1 positivity in the included studies ranges from $27 \%$ to $84 \%$. The target cell populations analyzed by immunohistochemistry in the included studies were immune cells (4 studies), tumour cells (3 studies), and immune cells or tumour cells (3 studies).

\section{Efficacy of PD-L1 As a Predictive Biomarker}

The included studies were divided into the $\geq 1 \%$ threshold, $\geq 5 \%$ threshold, and $\geq 25 \%$ threshold based, on the PDL1 expression threshold used in the individual studies. In all 3 groups, the PD-L1-positive patients had a better objective response than PD-L1-negative patients. A total of six studies reported ORR based on $\geq 1 \%$ PD-L1 threshold. 
TABLE I Studies included in the analysis

\begin{tabular}{|c|c|c|c|c|c|}
\hline Reference & $\begin{array}{c}\text { ClinicalTrials.gov } \\
\text { ID }\end{array}$ & Study drug & Dose & $\begin{array}{l}\text { Patients } \\
\text { (n) }\end{array}$ & $\begin{array}{c}\text { Newcastle- } \\
\text { Ottawa } \\
\text { scale }\end{array}$ \\
\hline \multicolumn{6}{|l|}{ Anti-PD-1 trials } \\
\hline Sharma et al., $2016^{23}$ & NCT01928394 & Nivolumab & 3 mg/kg IV every 2 weeks & 86 & 5 \\
\hline Balar et al., $2017^{24}$ & NCT02335424 & Pembrolizumab & 200 mg every 3 weeks & 370 & 6 \\
\hline Plimack et al., $2017^{10}$ & NCT01848834 & Pembrolizumab & $10 \mathrm{mg} / \mathrm{kg}$ IV every 2 weeks & 33 & 6 \\
\hline Sharma et al., $2017^{25}$ & NCT02387996 & Nivolumab & $\begin{array}{c}3 \mathrm{mg} / \mathrm{kg} \text {, } 1 \text {-hour IV infusion } \\
\text { every } 2 \text { weeks }\end{array}$ & 270 & 6 \\
\hline \multicolumn{6}{|l|}{ Anti-PD-L1 trials } \\
\hline Balar et al., $2017^{22}$ & NCT02108652 & Atezolizumab & 1200 mg IV every 3 weeks & 119 & 6 \\
\hline $\begin{array}{l}\text { Perez-Gracia et al., } 2017^{26}{ }^{\prime} \\
\quad \text { Rosenberg et al., } 2016^{21}\end{array}$ & NCT02108652 & Atezolizumab & 1200 mg IV every 3 weeks & 310 & 6 \\
\hline Powles et al., $2017^{27}$ & NCT01693562 & Durvalumab & $10 \mathrm{mg} / \mathrm{kg}$ every 2 weeks & 191 & 6 \\
\hline Pal et al., $2018^{28}$ & NCT02589717 & Atezolizumab & 200 mg IV every 3 weeks & 214 & 3 \\
\hline Patel et al., $2018^{29}$ & NCT01772004 & Avelumab & $10 \mathrm{mg} / \mathrm{kg}$ every 2 weeks & 249 & 6 \\
\hline Petrylak et al., $2018^{30}$ & NCT01375842 & Atezolizumab & 15 mg/kg every 3 weeks & 95 & 5 \\
\hline
\end{tabular}

a This study had 2 cohorts: first-line and second-line. The study reporting the most recent or complete data was used for the analysis. IV = intravenously.

TABLE II PD-L1 testing method and prevalence of PD-L1 positivity in the included studies

\begin{tabular}{|c|c|c|c|c|c|c|}
\hline \multirow[t]{2}{*}{ Reference } & \multicolumn{2}{|c|}{ PD-L1 assay } & \multirow{2}{*}{$\begin{array}{c}\text { Target } \\
\text { cell } \\
\text { population }\end{array}$} & \multicolumn{3}{|c|}{ PD-L1 expression (\%) } \\
\hline & Developer & $\begin{array}{l}\text { Antibody clone } \\
\text { for IHC }\end{array}$ & & Threshold & Positive & Negative \\
\hline \multicolumn{7}{|l|}{ Anti-PD-1 trials } \\
\hline Sharma et al., $2016^{23}$ & Dako & $28-8$ & TCs & $\geq 1$ & 37 & 63 \\
\hline \multirow[t]{2}{*}{ Balar et al., $2017^{24}$} & Agilent & $22 \mathrm{C} 3$ & TCs and ICs & $\geq 10$ & 30 & 70 \\
\hline & & & & $\geq 1$ & 83 & 17 \\
\hline Plimack et al., $2017^{10}$ & Dako & $22 \mathrm{C} 3$ & TCs and ICs & $\geq 1$ & 84 & 16 \\
\hline \multirow[t]{2}{*}{ Sharma et al., $2017^{25}$} & Dako & $28-8$ & TCs & $\geq 1$ & 46 & 54 \\
\hline & & & & $\geq 5$ & 31 & 69 \\
\hline \multicolumn{7}{|l|}{ Anti-PD-L1 trials } \\
\hline \multirow[t]{2}{*}{ Balar et al., $2017^{22}$} & Ventana & SPI-142 & $\mathrm{ICs}$ & $\geq 1$ & 67 & 33 \\
\hline & & & & $\geq 5$ & 27 & 73 \\
\hline \multirow[t]{2}{*}{ Perez-Gracia et al., 2017²6 } & Ventana & SP-142 & $\mathrm{ICs}$ & $\geq 1$ & 67 & 33 \\
\hline & & & & $\geq 5$ & 32 & 68 \\
\hline Powles et al., $2017^{27}$ & Ventana & SP-263 & ICs or TCs & $\geq 25$ & 55 & 45 \\
\hline Pal et al., $2018^{28}$ & Ventana & SP-142 & $\mathrm{ICs}$ & $\geq 5$ & 52 & 48 \\
\hline Patel et al., $2018^{29}$ & Dako & $73-10$ & TCs & $\geq 5$ & 45 & 55 \\
\hline Petrylak et al., $2018^{30}$ & Ventana & SPI-142 & $\mathrm{ICs}$ & $\geq 5$ & 53 & 7 \\
\hline
\end{tabular}

$\mathrm{IHC}=$ immunohistochemistry; TCs = tumour cells; ICs = immune cells.

A statistically significant difference was observed in ORR between PD-L1-positive and PD-L1-negative patients (OR: $1.74 ; 95 \%$ CI: 1.20 to $2.53 ; p=0.00$ ), favouring PD-L1-positive patients [Figure 2(A)]. Six studies reporting ORR based on $>5 \%$ PD-L1 threshold were included for the analysis. Three studies reported ORR based on both the study thresholds.
A statistically significant difference was observed in ORR between PD-L1-positive and PD-L1-negative patients (OR: 2.74 ; 95\% CI: 2.01 to $3.724 ; p<0.00$ ), favouring PD-L1positive patients [Figure 2(C)]. Only one study reported PD-L1 expression based on a $\geq 25 \%$ threshold, with a statistically significant ORR favouring PD-Ll positivity (OR: 
7.13; 95\% CI: 2.38 to 21.40). A fixed-effects model was used for analysis given that the $I^{2}$ was $<50 \%$.

\section{Threshold of PD-L1 Expression in Predicting Positive Therapeutic Response}

Sensitivity, specificity, positive predictive value, and negative predictive value were used to assess the thresholds. $A \geq 1 \%$ threshold of PD-L1 expression was associated with high sensitivity (73.04\%) and low specificity (39.62): the reverse was true for a $\geq 5 \%$ threshold. Similarly, a $\geq 1 \%$ threshold of PD-Ll expression was associated with a relatively low positive predictive value (1.21), and the

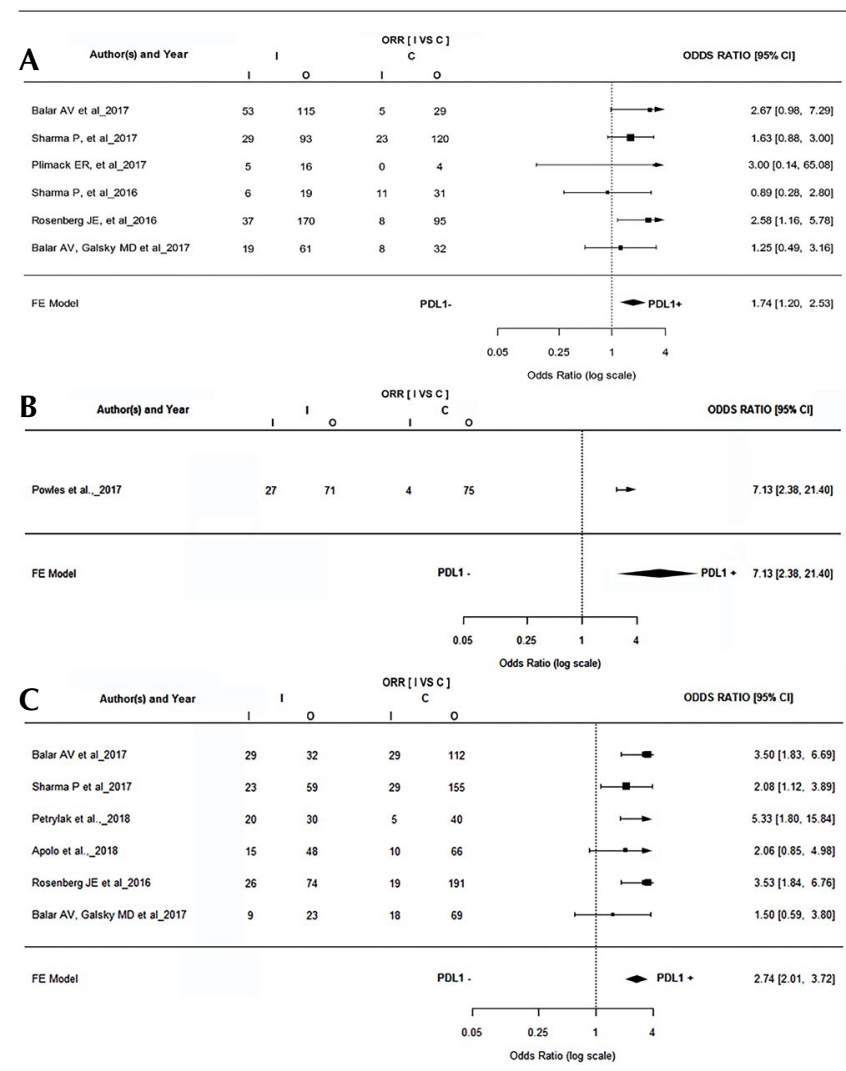

FIGURE 2 (A) Forest plot shows that patients with higher PD-L1 positivity (PD-L1 expression threshold of $\geq 1 \%$ ) responded better to treatment. (B) Forest plot shows that patients with higher PD-L1 positivity (PD-L1 expression threshold of $\geq 25 \%$ ) responded better to treatment. (C) Forest plot shows that patients with higher PD-L1 positivity (PD-L1 expression threshold of $\geq 5 \%$ ) responded better to treatment. ORR = objective response rate; $\mathrm{I}=$ intervention; $\mathrm{C}=$ control; $\mathrm{Cl}=$ confidence interval; $F E=$ fixed effects.

TABLE III Diagnostic characteristics of the three PD-L1 thresholds

\begin{tabular}{|c|c|c|c|c|c|c|}
\hline \multirow[t]{2}{*}{ Characteristic } & \multicolumn{6}{|c|}{ PD-L1 threshold (\%) } \\
\hline & $\geq 1 \%$ positivity & $95 \% \mathrm{Cl}$ & $\geq 5 \%$ positivity & $95 \% \mathrm{Cl}$ & $\geq 25 \%$ positivity & $95 \% \mathrm{Cl}$ \\
\hline Sensitivity & 73.04 & 66.4 to 79 & 52.59 & 45.95 to 59.16 & 87.10 & 70.17 to 96.37 \\
\hline Specificity & 39.62 & 36.18 to 43.14 & 70.41 & 67.31 to 73.38 & 51.37 & 42.97 to 59.72 \\
\hline Positive predictive value & 1.21 & 1.09 to 1.34 & 1.78 & 1.52 to 2.08 & 1.79 & 1.44 to 2.22 \\
\hline Negative predictive value & 0.68 & 0.53 to 0.87 & 0.67 & 0.58 to 0.78 & 0.25 & 0.10 to 0.64 \\
\hline
\end{tabular}

negative predictive value ( 0.68 and 0.67 ) was high for both the thresholds. Detailed results are provided in Table III. Further, we compared the oR for the two thresholds and found that $\geq 5 \%$ PD-L1 expression is better in predicting objective response $(p<0.0001)$ in patients with urothelial cancer treated with PD-1/PD-L1 drugs.

\section{Subgroup Analysis with Testing Platforms}

Dako and Ventana were the most frequently used diagnostic platforms. A total of three studies used the Dako platform with $\geq 1 \%$ as the threshold. The pooled on was found to be 1.34 (95\% CI: 0.88 to 2.04). Similarly, two studies that used the Dako platform reported a PD-L1 threshold of $\geq 5 \%$ with a pooled on of 1.79 (95\% CI: 1.20 to 2.67), which was statistically significant ( $p=0.0045$ ). Studies that used the Ventana platform also used $\geq 1 \%$ and $\geq 5 \%$ as thresholds, with a pooled oR of 1.66 (95\% CI: 0.99 to 2.78$)$ and 3.05 (95\% CI: 1.89 to 4.92 ) respectively (Figure 3 ).

\section{Clinical Efficacy and Safety of PD-1 vs. PD-L1 Drugs}

A total of six and four studies included patients treated with PD-L1 and PD-1 inhibitors respectively. The ORR ranged from $19.6 \%$ to $24.4 \%$ for PD-1 inhibitors and from $15 \%$ to $26 \%$ for PD-L1 inhibitors. Similarly, PFS ranged from 2 to 2.8 months for PD-1 drugs and 1.5 to 6.3 for PD-L1 drugs. Comparison of overall efficacy in terms of ORR $(p=0.02)$, PFS $(p=0.52)$, and os $(p=0.48)$ revealed no statistically significant difference between the groups, although PD-1 drugs had a better efficacy with respect to ORR, PFS, and os in the included studies (Figure 4). In terms of safety, PD-L1 drugs had a better safety profile, with statistically significant differences observed between any treatment-related adverse event (AE) $(p=0.09)$, treatment-related grade 3 or greater $\mathrm{AE}(p=0.01)$, treatment-related serious $\mathrm{AE}(p=0.00)$, and pruritis $(p<0.00)$.

\section{Publication Bias}

Visual inspection of funnel plots constructed with ORR for $\geq 1 \%$ and $\geq 5 \%$ threshold did not reveal substantial asymmetry, suggestive of relatively little publication bias (Figure 5).

\section{DISCUSSION}

Recent phase III trials with PD-1/PD-L1 inhibitors in urothelial cancer revealed a limited role for PD-L1 expression in predicting a favourable therapeutic response $\mathrm{e}^{20}$. Because FDA approval for the PD-L1 diagnostic assays as companion or complimentary tests was based on preliminary phase I/II evidence, we performed a meta-analysis to re-ascertain the

$\mathrm{Cl}=$ confidence interval. 


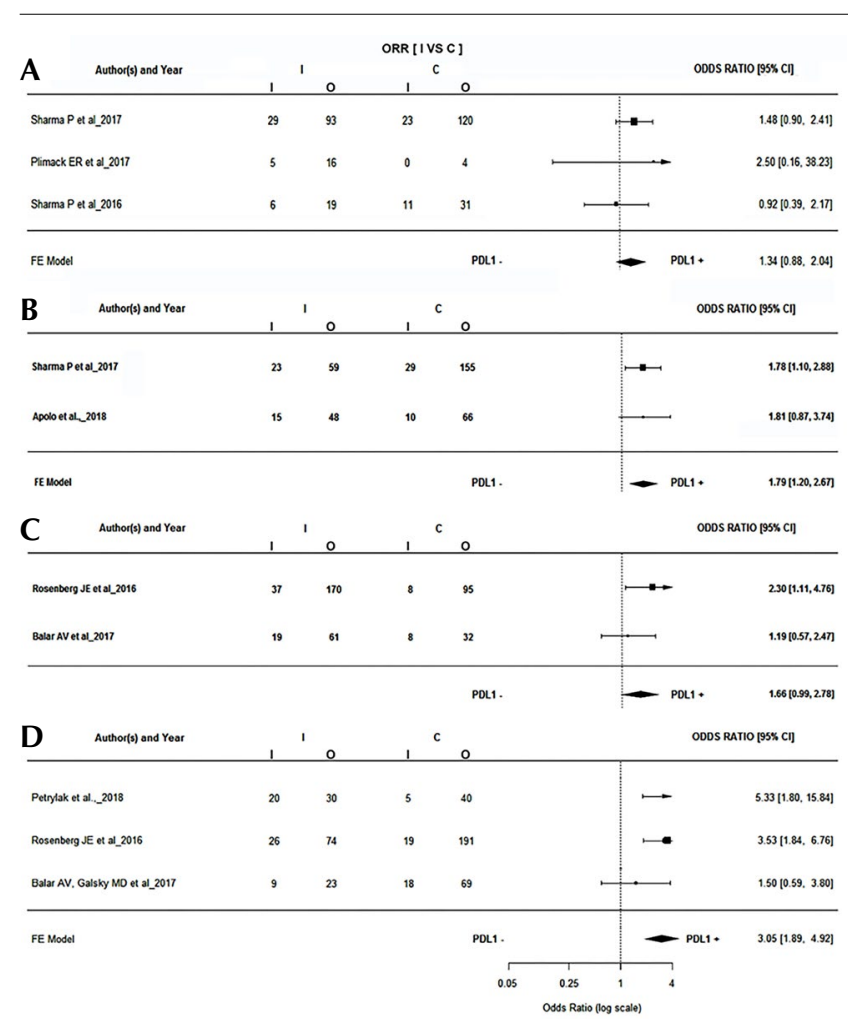

FIGURE 3 Forest plots for response rates based on PD-L1 detection platform. (A) Dako Corporation (Agilent Technologies, Santa Clara, CA, U.S.A.), $\geq 1 \%$ threshold, (B) Dako, $\geq 5 \%$ threshold, (C) Ventana (Roche Diagnostics, Risch-Rotkreuz, Switzerland), $\geq 1 \%$ threshold, (D) Ventana, $\geq 5 \%$ threshold. ORR = objective response rate; $\mathrm{I}=$ intervention; $\mathrm{C}=$ control; $\mathrm{Cl}=$ confidence interval; $\mathrm{FE}=$ fixed effects.

potential of PD-L1 expression levels for biomarker-guided pharmacotherapy. Despite the wide variation in the diagnostic and interpretive methods used in the included studies, our analysis revealed PD-L1 expression-positive patients to be more likely to experience a positive treatment response than PD-L1-negative patients with PD-1/ PD-Ll drugs.

Out of the ten studies that were included for analysis, four studies were conducted with atezolizumab, two with nivolumab and pembrolizumab, and one each with avelumab and durvalumab. The PD-L1 expression threshold, assay platform, target cell population, and nature of biopsy samples used were also different in the included studies. While seven studies were conducted in previously cisplatinor chemotherapy-treated patients, three were conducted in a first-line setting in cisplatin-ineligible patients. Although there were differences in PD-Ll expression testing, two studies using the same assay with the same drug (atezolizumab) in first- and second-line settings revealed contrasting results. While the expression of PD-L1 seems to better predict for objective response in second-line trials with atezolizumab ( $18 \%$ vs. $8 \%$ ), in a first-line setting there was no difference in efficacy $(24 \% \text { vs. } 21 \%)^{21,22}$. In order to better understand the beneficial role of PD-L1 expression in first-line settings, the ongoing KEYNOTE-361 study (NCT02853305 at https://ClinicalTrials.gov/) is assessing the efficacy of pembrolizumab in combination with

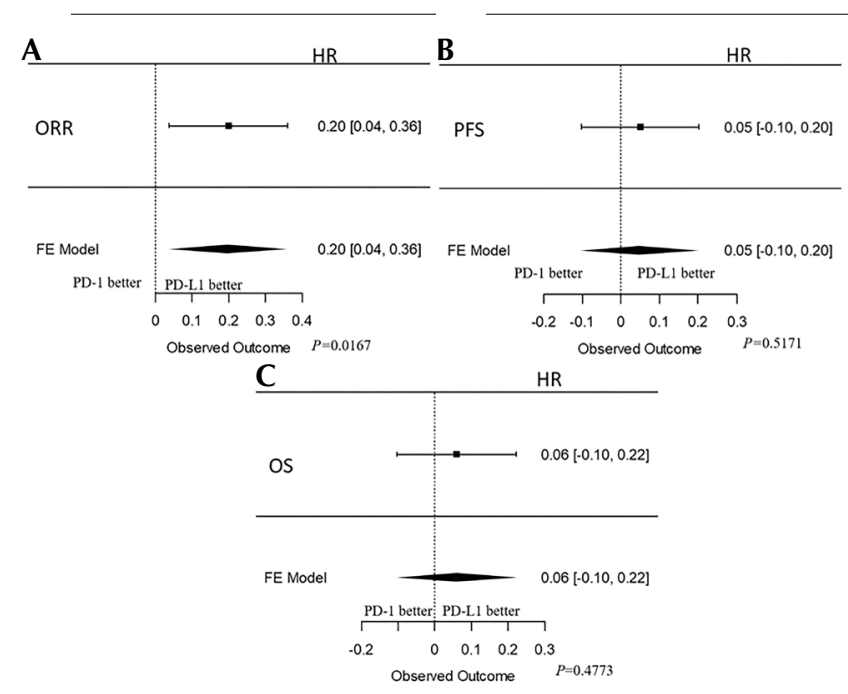

FIGURE 4 Forest plots showing the efficacy of anti-PD-1 and anti-PDL1 drugs. (A) Objective response rate (ORR). (B) Progression-free survival (PFS). (C) Overall survival (OS). HR = hazard ratio; FE = fixed effects.
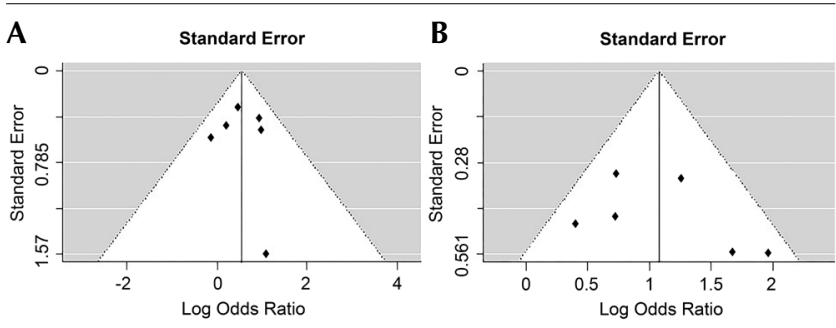

FIGURE 5 (A) Publication bias for objective response rate, PD-L1 $\geq 1 \%$ threshold. (B) Publication bias for objective response rate, PD-L1 $\geq 5 \%$ threshold.

chemotherapy compared with chemotherapy alone in patients with positive and negative PD-Ll expression in a first-line setting. In the first-line setting, atezolizumab in combination with platinum-based therapy has also proven to be effective in extending PFS in comparison with chemotherapy alone ${ }^{31}$. Based on the findings from these studies, the FDA has limited the use of atezolizumab and pembrolizumab in first-line settings to patients with positive PD-Ll expression, which further substantiates the role of PD-L1 expression testing.

A higher threshold was found to have a better prognostic ability in lung cancer settings ${ }^{32}$. One study that used a $\geq 25 \%$ threshold revealed a sensitivity of $87 \%$, but the specificity was only $51 \%$. The expression of PD-L1 in the urothelial cancer microenvironment has been relatively low. The low expression of PD-L1 is further complicated by the use of archaic biopsy samples for immunohistochemistry, which give deceptive results. In a few studies, PD-L1 expression was determined at baseline; other studies estimated PD-Ll expression during the study progression, with archaic samples.

The nature of PD-Ll testing kits and platforms, together with the samples, determines the overall effectiveness of PD-L1 testing. In our study, the included articles 
predominantly used Dako and Ventana platforms for PD-Ll testing. To confirm the uniformity of our overall findings, we performed a subgroup analysis with only those studies that used the Dako and Ventana platforms separately. In both the cases, the results were in agreement with our overall results favouring $\geq 5 \%$ as the threshold. But these results need to be interpreted with caution because we did not account for the different antibodies used with the Dako and Ventana platforms. Statistical significance was observed only with $\geq 5 \%$ favouring PD-L1 positive expression, which suggests that a higher threshold could balance for variation in the assay method.

Based on our analysis, PD-L1 expression status is a good prognostic marker for predicting clinical efficacy. A previous meta-analysis by Fan et al. that included six single-arm studies of patients with urothelial cancer reported the $\geq 5 \%$ PD-L1 expression threshold to be a better indicator for therapeutic response than the $\geq 1 \%$ threshold. The researchers also observed that patients with low PD-L1 expression had a better objective response with PD-1 drugs than with PDL1 drugs. This could be due to the fact that PD-1 could also be activated by ligands other than PD-L1, and hence the activity of PD-1 drugs could be independent of PD-L1 expression. Further studies are required to confirm these hypotheses ${ }^{33}$. However, the study by Fan et al. did not account for the differences in the assay methodology. The results of our analysis also indicate that $\mathrm{a} \geq 5 \% \mathrm{PD}$-L1 threshold has a better predictive ability for ORR. But $\mathrm{a} \geq 5 \%$ threshold was also associated with relatively low sensitivity and high specificity. This suggests that using this criterion for PD-L1 positive status may cause us to miss recruiting patients capable of eliciting a positive therapeutic response. A previous study by Balar et al. with pembrolizumab also revealed a higher threshold to be associated with lower sensitivity and higher positive predictive value ${ }^{13}$. In addition, PD-1/PDL1 drug therapy carries its own economic burden on the urothelial cancer community. A lower threshold might be beneficial to obtain an optimum benefit for a maximum of patients. Further improving the methods and establishing a common standardized platform for expression assays might improve the usefulness of PD-L1 expression levels as predictive markers.

The target cell population for analysis of PD-L1 expression is also widely debated. Each kit has its own recommended target-cell population within the tumour microenvironment. A standardization for target-cell populations should be performed with each cancer type since PD-L1 expression in tumour and immune cells depends on the type of cancer ${ }^{34}$. The articles included in the current study used tumour cells, immune cells, or both as the target cell population for PD-L1 detection.

All the studies included for this meta-analysis were early, single-arm studies used for the systematic appraisal of evidence. We did not include the KEYNOTE-045 and IMvigor phase III randomized controlled trials (RCTs), which compared PD-1/PD-L1 drugs with chemotherapy, for our evidence synthesis. These studies were not included because combining single-arm studies with RCTs for statistical synthesis might confound the overall summary effect estimate between PD-L1-positive and PD-L1-negative patients. Based on the results from the current study,
PD-L1 expression enriches the clinical response and might be considered to be a surrogate marker for predicting efficacy of PD-1/PD-L1 drugs. The predictive ability of PDL1 expression could be optimized by selecting appropriate target cell populations and cut-off values. But the clinical utility of PD-L1 testing in urothelial carcinoma has to be ascertained in well-planned clinical studies.

Due to limited data with $\geq 10 \%$ thresholds, the possibility of a higher threshold with better predictive power cannot be ruled out. Given that phase III RCTs with PD-1/PD-L1 drugs in patients with urothelial cancer are ongoing, a comprehensive landscape of PD-L1 expression levels in urothelial cancer could be analyzed with the results of the ongoing trials. Further, due to the unavailability of survival endpoint data, we have used objective response, which might have its own intrinsic limitations in predicting efficacy.

\section{CONCLUSIONS}

To conclude, PD-L1 expression could be used in patients with urothelial cancer as a biomarker for favourable therapeutic response. We suggest a threshold of $5 \%$ or greater as the cut-off to predict clinical response although it should be used with caution. Future clinical trials comparing PD-1/PD-L1 drugs with standard-of-care therapy should include PD-L1-positive patients to evaluate efficacy. Further, methods other than immunohistochemistry should be assessed and used in clinical trials for PD-L1 expression analysis. Other potential markers might to be explored to identify patients with potential clinical response to PD-1/ PD-L1 drugs. Randomized controlled trials with randomization based on PD-L1 expression levels are required to gain further insights. The results of the meta-analysis will help in fine-tuning patient recruitment for future clinical trials involving anti PD-1/PD-L1 antibodies either alone or in combination with other standard-of-care therapies.

\section{ACKNOWLEDGMENTS}

The authors thank the medical diagnosis team at AstraZeneca for their scientific comments on this manuscript. The authors acknowledge medical writing assistance, under the guidance of authors, rendered by Drs. Kaushik Subramanian, Anuradha Nalli, and Amit Bhat from Indegene Pvt. Ltd., Bangalore, India, and funded by AstraZeneca. The funders had no role in the design and interpretation of the study.

\section{CONFLICT OF INTEREST DISCLOSURES}

We have read and understood Current Oncology's policy on disclosing conflicts of interest, and we declare that we have none.

\section{AUTHOR AFFILIATIONS}

*Department of Urology, Sun Yat-Sen Memorial Hospital, Sun YatSen University, Guangzhou, and ${ }^{\dagger}$ Department of Pathology, The First Affiliated Hospital, College of Medicine, Zhejiang University, Hangzhou, P.R.C.

\section{REFERENCES}

1. Antoni S, Ferlay J, Soerjomataram I, Znaor A, Jemal A, Bray F. Bladder cancer incidence and mortality: a global overview and recent trends. Eur Urol 2017;71:96-108.

2. Dietrich B, Srinivas S. Urothelial carcinoma: the evolving landscape of immunotherapy for patients with advanced disease. Res Rep Urol 2018;10:7-16. 
3. Approved Drugs - Atezolizumab for Urothelial Carcinoma. US FDA. [Available online at: https://www.fda.gov/drugs/ informationondrugs/approveddrugs/ucm501878.htm; cited 12 December 2018]

4. Approved Drugs - Pembrolizumab (Keytruda): Advanced or Metastatic Urothelial Carcinoma. US FDA. [Available online at: https://www.fda.gov/Drugs/InformationOnDrugs/ ApprovedDrugs/ucm559300.htm; cited 12 December 2018]

5. Approved Drugs - Durvalumab (Imfinzi). US FDA. [Available online at: https://www.fda.gov/drugs/informationondrugs/ approveddrugs/ucm555930.htm; cited 12 December 2018]

6. Approved Drugs - Nivolumab for Treatment of Urothelial Carcinoma. US FDA. [Available online at: https://www.fda.gov/ Drugs/InformationOnDrugs/ApprovedDrugs/ucm539646. htm; cited 12 December 2018]

7. Approved Drugs - FDA grants accelerated approval to avelumab for urothelial carcinoma. [Available online at: https:// www.fda.gov/Drugs/InformationOnDrugs/ApprovedDrugs/ ucm557162.htm; cited 12 December 2018]

8. Chen DS, Mellman I. Oncology meets immunology: the cancer-immunity cycle. Immunity 2013;39:1-10.

9. ZhuJ,ArmstrongAJ,FriedlanderTW, etal. Biomarkers ofimmunotherapyin urothelial and renal cell carcinoma:PD-L1, tumor mutational burden, and beyond. J Immunother Cancer 2018; 6:4.

10. Plimack ER, Bellmunt J, Gupta S, et al. Safety and activity of pembrolizumab in patients with locally advanced or metastatic urothelial cancer (KEYNOTE-012): a non-randomised, open-label, phase 1b study. Lancet Oncol 2017;18:212-20.

11. Balar AV, Castellano DE, O'Donnell PH, et al. Pembrolizumab as first-line therapy in cisplatin-ineligible advanced urothelial cancer: results from the total KEYNOTE-052 study population. JClin Oncol 2017;35. [Available online at: http:// www.embase.com/search/results? subaction=viewrecord \&from=export\&id=L618006643]

12. U.S. Food and Drug Administration. Summary of safety and effectiveness data (SSED) for PD-L1 IHC 28-8 pharmDx. [Available online at: http://www.accessdata.fda.gov/cdrh_docs/ pdf15/p150025b.pdf]

13. U.S. Food and Drug Administration. Summary of safety and effectiveness data (SSED) for PD-L1 IHC 22C3 pharmDx. [Available online at: https://www.accessdata.fda.gov/cdrh_docs/ pdf15/P150013S006b.pdf]

14. U.S. Food and Drug Administration. Summary of safety and effectiveness data (SSED) for VENTANA PD-L1 (SP142) Assay. [Available online at: http://www.accessdata.fda.gov/ cdrh_docs/pdf16/p160006b.pdf]

15. Hersom M, Jørgensen JT. Companion and complementary diagnostics-focus on PD-L1 expression assays for PD-1/ PD-L1 checkpoint inhibitors in non-small cell lung cancer. Ther Drug Monit 2018;40:9-16.

16. ASCO POST. FDA restricts use of pembrolizumab or atezolizumab to treat urothelial cancer due to efficacy concerns in some patients. [Available online at: http://www.ascopost.com/ News/59004]

17. Powles T, Durán I, Heijden MS van der, et al. Atezolizumab versus chemotherapy in patients with platinum-treated locally advanced or metastatic urothelial carcinoma (IMvigor211): a multicentre, open-label, phase 3 randomised controlled trial. Lancet 2018;391:748-57.

18. Moher D, Liberati A, Tetzlaff J, Altman DG, PRISMA Group. Preferred reporting items for systematic reviews and metaanalyses: the PRISMA statement. PLoS Med 2009;6:e1000097.
19. Xing D, Xu Y, Liu Q, et al. Osteoarthritis and all-cause mortality in worldwide populations: grading the evidence from a meta-analysis. Sci Rep 2016;6:24393.

20. Bellmunt J, de Wit R, Vaughn DJ, et al. Pembrolizumab as second-line therapy for advanced urothelial carcinoma. N Engl J Med 2017;376:1015-26.

21. Rosenberg JE, Hoffman-Censits J, Powles T, et al. Atezolizumab in patients with locally advanced and metastatic urothelial carcinoma who have progressed following treatment with platinum-based chemotherapy: a single-arm, multicentre, phase 2 trial. Lancet 2016;387:1909-20.

22. Balar AV, Galsky MD, Rosenberg JE, et al. Atezolizumab as first-line treatment in cisplatin-ineligible patients with locally advanced and metastatic urothelial carcinoma: a single-arm, multicentre, phase 2 trial. Lancet 2017;389:67-76.

23. Sharma P, Callahan MK, Bono $\mathrm{P}$, et al. Nivolumab monotherapy in recurrent metastatic urothelial carcinoma (CheckMate 032): a multicentre, open-label, two-stage, multi-arm, phase 1/2 trial. Lancet Oncol 2016;17:1590-98.

24. Balar AV, Castellano D, O'Donnell PH, et al. First-line pembrolizumab in cisplatin-ineligible patients with locally advanced and unresectable or metastatic urothelial cancer (KEYNOTE-052): a multicentre, single-arm, phase 2 study. Lancet Oncol 2017;18:1483-92.

25. Sharma P, Retz M, Siefker-Radtke A, et al. Nivolumab in metastatic urothelial carcinoma after platinum therapy (CheckMate 275): a multicentre, single-arm, phase 2 trial. Lancet Oncol 2017;18:312-22.

26. Perez-Gracia JL, Loriot Y, Rosenberg JE, et al. Atezolizumab in platinum-treated locally advanced or metastatic urothelial carcinoma: outcomes by prior number of regimens. Eur Urol 2018;73:462-68.

27. Powles T, O'Donnell PH, Massard C, et al. Efficacy and safety of durvalumab in locally advanced or metastatic urothelial carcinoma: updated results from a phase 1/2 open-label study. JAMA Oncol 2017;3:e172411.

28. Pal SK, Hoffman-Censits J, Zheng H, Kaiser C, Tayama D, Bellmunt J. Atezolizumab in platinum-treated locally advanced or metastatic urothelial carcinoma: clinical experience from an expanded access study in the United States. Eur Urol 2018;73:800-6.

29. Patel MR, Ellerton J, Infante JR, et al. Avelumab in metastatic urothelial carcinoma after platinum failure (JAVELIN Solid Tumor): pooled results from two expansion cohorts of an open-label, phase 1 trial. Lancet Oncol 2018;19:51-64.

30. Petrylak DP, Powles T, Bellmunt J, et al. Atezolizumab (MPDL3280A) monotherapy for patients with metastatic urothelial cancer long-term outcomes from a phase 1 study. JAMA Oncol 2018;4:537-44.

31. Galsky MD, Arija JÁA, Bamias A, et al. Atezolizumab with or without chemotherapy in metastatic urothelial cancer (IMvigor130): a multicentre, randomised, placebo-controlled phase 3 trial. Lancet 2020;395:1547-57.

32. Fehrenbacher L, Spira A, Ballinger M, et al. Atezolizumab versus docetaxel for patients with previously treated non-smallcell lung cancer (POPLAR): a multicentre, open-label, phase 2 randomised controlled trial. Lancet 2016;387:1837-46.

33. Liu J, Zhang C, Hu J, et al. Effectiveness of anti-PD-1/PD-L1 antibodies in urothelial carcinoma patients with different PD-L1 expression levels: a meta-analysis. Oncotarget 2018;9:12400-7.

34. Wang X, Teng F, Kong L, Yu J. PD-L1 expression in human cancers and its association with clinical outcomes. Onco Targets Ther 2016;9:5023-39. 